\section{Digestive \\ Surgergiry}

Abdominoperineal resection 32

Acute gastric volvulus 169

- pancreatitis 336

Ampulla of Vater 262

Anastomosis 38, 283

-, pancreatic 296

Anastomotic dehiscence, leakage, disruption 38

Angiodysplasia 387

Angioembolization 224

Angiogenesis 331

Angioplasty 358

Aortic valve stenosis 387

Bariatric surgery 1

Benign bile duct strictures 304

Bile reflux 325

Biliary injury 370

Bleeding 217

Blood loss 32

Bovine pericardium 283

Bowel cleansing 375

- irrigation 375

Branch-duct-oriented resection 121

Bronchogenic cyst 209

Budd-Chiari syndrome 358

CA125 209

Carcinoma 313

Cholangiocarcinoma, CA 19-9 level 319

Chyle leakage 86

- -, complications 86

Circular stapler 23

Cirrhosis 103

Colon perforation 303

Colorectal carcinoma 80

- surgery 110, 375

Completion pancreatectomy, indications 229

- $\quad$, postoperative outcome 229

- -, survival data 229

Complications, pancreaticoduodenectomy 203

Crohn's disease 241, 346

- - activity index 241

Curative resection 313

Cystadenocarcinoma 186

Cystadenoma 186

D2 gastrectomy 192

- lymphadenectomy 192

- -, morbidity 192

- $\quad$, mortality 192
Delayed closure 150

Digestive tract schwannoma 265

Distal/subtotal pancreatectomy 229

Duct stenosis, pancreatic 250

Duodenal perforation 150

Duodenostomy 150

Early hemorrhage 203

Ectopic pancreas 262

Embryo 186

Emergency surgery 217

Endoscope-assisted surgery 209

Endoscopic retrograde cholangiopancreatography 150, 250

Enteral nutrition 336

- - , total 139

- stent 28

Epidermal growth factor receptor 74

Esophageal cancer 86, 164

- cyst 209

- surgery 159

Esophagectomy 86

Fecal incontinence 146

$\alpha$-Fetoprotein 103

Fistula, pancreatic 250

Foley catheter 150

Foregut intubation 292

Gastric cancer 28, 192, 217

- cardia 313

- carcinoma, prognosis 313

- schwannoma 265

Gastrointestinal bleeding 387

- schwannoma 265

- stromal tumours 265

Gonadal epithelium 186

Graft survival 65

Haemorrhage 224

Hepatectomy 80, 103, 115

Hepatic functional mass 44

- resection, partial 139

- vein thrombosis 358

Hepatocellular carcinoma 103, 235

Heyde syndrome 387

Hiatus hernia 169

Hydatid cyst 198

Ileal J pouch 23

Ileocecal resection 346

Ileocolic resection 346

In situ trimming 115
India ink tattooing 303

Infections 336

Inferior vena cava reconstruction 115

Intestine 38

Intraductal papillary mucinous tumors, pancreas 121

Intraoperative peritoneal hyperthermic chemotherapy 93

Intrinsic clearance of antipyrine 44

Jejunal pouch reconstruction 381

Joint symptoms 60

Laparoscopic cholecystectomy 198, 370

- gastric tube formation 164

- intervention 110

- resection 346

Laparoscopy 164, 283

Linear stapler 23

Lithotomy position 32

Liver 186

- function 139

- mass 198

- metastasis 80

- regeneration 44

Local excision 12

Long-term results, benign biliary strictures 304

Lymph node metastasis 80

Lymphadenectomy, extent 192

Malignant gastric outlet obstruction, non-resectable 28

Mesenteric schwannoma 265

Mesorectal excision 51

Minimal pancreatectomy 121

Morphological regeneration 44

Mortality 336

Neurogenic gastro-intestinal tumours 265

Neutrophil 179

Nutrition, perioperative 139

Nutritional assessment 159

Obesity, surgery 1

Oesophagitis 325

Oesophagogastric surgery 292

Open transthoracic esophagectomy 164

Operative time 32

Ovarian stroma 186

Palliation 28

Pancreas 186, 250 
Pancreatic adenocarcinoma 74

- anastomosis 203

- cancer 28

- surgery 203

Pancreaticoduodenectomy 203, 224, 296

Pancreaticojejunostomy 296

Pancreatoduodenectomy 229

Parenteral nutrition 139, 336

PD complications 224

Pediatric liver transplantation 65

Perforation 217

Periampullary tumor 262

Peritoneal metastases 93

Pharyngostomy 292

Polyester vascular prosthesis 115

Portal aneurysm 259

- vein chemotherapy 235

Portosystemic shunts 358

- venous shunt 259

Postcholecystectomy injury 304

Postoperative complications 179

- infectious complications 159

Pouchitis 60

Preoperative bowel preparation 375

- rectal carcinoma 173

Primary sclerosing cholangitis 319

- skin closure 255

Prognosis 93

Proximal gastrectomy 325, 381

Pseudocyst, pancreatic 250
Radical gastrectomy 192

Radiofrequency coagulation 146

Reconstruction 370

Rectal adenoma 12

- cancer 12, 51, 331

- -, complications 51

- -, recurrence 51

- carcinoma 173

- -, recurrence 173

- - , short-term preoperative radiotherapy 173

- prolapse 146

Reflux esophagitis 381

Restorative proctocolectomy 60

Right lateral position 32

Risk indicators for intra-/postoperative complications 110

Sentinel bleed 224

Shunt closure 259

Shunts 358

Sphincterotomy 262

Spilled gall stones 198

Staple line reinforcement 283

Stenting, pancreatic 250

Steroids 60

Stoma closure 255

Stomach neoplasm 93

Surgeons and rectal surgery 51

Surgery 241

Surgical stress 179
Technical-variant graft 65

Thiersch's procedure 146

Thoracoscopic surgery 209

Thoracotomy 164

Thymidine phosphorylase 331

Tissue microarray 74

Total ampullectomy 262

- gastrectomy 325, 381

- mesorectal excision 51

Transanal endoscopic microsurgery 12

Transarterial chemoembolization 235

Transjugular intrahepatic portosystemic shunt 358

Ulcerative colitis 60,179

Vascular endothelial growth factor 331

- injury 370

Vienna classification 241

Wound infection 255 